\title{
Low Frequency Parasitic Effects in RF Transistors and their Impact on Power Amplifier Performances
}

\author{
Raymond Quéré, Raphael Sommet, Philippe Bouysse, Tibault Reveyrand, Denis Barataud, Jean Pierre Teyssier, \\ Jean Michel Nébus. \\ $\mathrm{XLIM} / \mathrm{C} 2 \mathrm{~S} 2$ \\ CNRS-University of Limoges \\ Limoges-France \\ raymond.quere@xlim.fr
}

\begin{abstract}
In this paper Low Frequency (LF) parasitic effects are assessed through three kinds of measurements. It is shown that LF S-parameters measurements allow to extract the thermal impedance of Heterojunction Bipolar Transistors (HBTs) and to put dispersive effects of AlGaN/GaN High Electron Mobility Transistors (HEMTs) into evidence. Large signal (RF pulsed and two tone intermodulation) confirm the impact of those parasitic effects on performances of Power Amplifiers.
\end{abstract}

Keywords-thermal, parasitic; traps; pulses; two-tone intermodulation.

\section{INTRODUCTION}

RF power technologies ( $\mathrm{Si}, \mathrm{GaAs}$ and $\mathrm{GaN}$ ) are either well established or very promising for future equipment in the defense and space domains. Notably the unique properties of $\mathrm{GaN}$ Field Effect Transistors in terms of breakdown voltage associated to high cut-off frequencies make possible a breakthrough for the development of new Solid State Power Amplifiers (SSPA) for Radars and Telecommunications applications. In both domains those SSPA are fed by modulated signals such as versatile pulses in the case of Radars or highly modulated signals in the case of telecommunication applications. Therefore it is essential to assess the dynamical behavior of RF devices fed by large modulated signals. Contrary to $\mathrm{CW}$ conditions, the dynamics of parasitic created by trapping and thermal effects can no longer be ignored. In order to get insights into the impact of those effects on large signal characteristics, a set of small and large signal measurements must be performed on transistors coming from various foundries. Those measurements are carried out through low frequency Sparameters measurements, time domain pulsed load-pull measurements as well as two-tone intermodulation measurements.

Thermal effects in bipolar transistors are very severe in Heterojunction Bipolar Transistors (HBTs) as well as in AlGaN/GaN HEMTs. Many techniques allow to measure the thermal impedance of RF power transistors. However thoses techniques are often based on complex measurements that necessitate a special preparation of the devices under test. If available an electrical measurement technique would provide a convenient mean to get data for determining nonlinear electrothermal models.

Parasitic effects in AlGaN/GaN HEMTs have been extensively studied both from the point of view of thermal effects and trapping effects. Many effects have been identified through the use of electrical methods such as Deep Level Transient Spectroscopy(DLTS) [1] or pulsed measurements initially on GaAs FETs [2] then on GaN devices [3]. Meanwhile a number of authors have conducted a number of studies about the physical mechanisms involved in the trapping process [4-6]. It appears from these studies that the trapping process is a very highly nonlinear process. This nonlinear process results from the dissymmetry of the time constants involved in carrier capture and emission processes. Indeed, in the case of deep levels, the time constant of the capture process falls into the nanosecondsmicroseconds range while that of the emission process falls into the microsecond-millisecond range. This effect has been accounted for in a number of circuit models which all represent the dissymmetry of the trapping [7-10]. From another side a number of linear models have been proposed, but they fail to accurately predict the large signal behavior [11 ]of the device. Recently a simplified model able to take into account both the drain lag and the gate lag was proposed and extensively tested in a Continuous Wave load-pull set up. The main result obtained from the measurements and confirmed by simulations with the model is that, in class $A B$ mode and with loads close to optimum ones, the average drain current decreases when the input power increases. This behavior can only be explained by trapping-detrapping of carriers either at the surface of the device or in the GaN layer. This behavior will be confirmed by measurements on modulated signals.

\section{TEST BENCHES DESCRIPTION}

Measurements were performed on three different test benches. The first one is a pulsed Large Signal Network Analyzer (LSNA), the second one is an Envelope measurement system. Large Signal Time Domain Pulsed Measurements and the third one is a LF S-parameters measurement set-up. 


\section{A. Time-domain measurement set up}

The time-domain measurements were performed, using the set-up shown at fig-1 which allow versatile time domain measurements in various pulsed configurations [12, 13]. In this case the measurements were performed on an $8 \times 75 \times 0.25$ $\mu \mathrm{m}^{2} \mathrm{AlGaN} / \mathrm{GaN}$ HEMT at the carrier frequency of $6 \mathrm{GHz}$ in different pulse configurations. A DC bias was applied to the device.

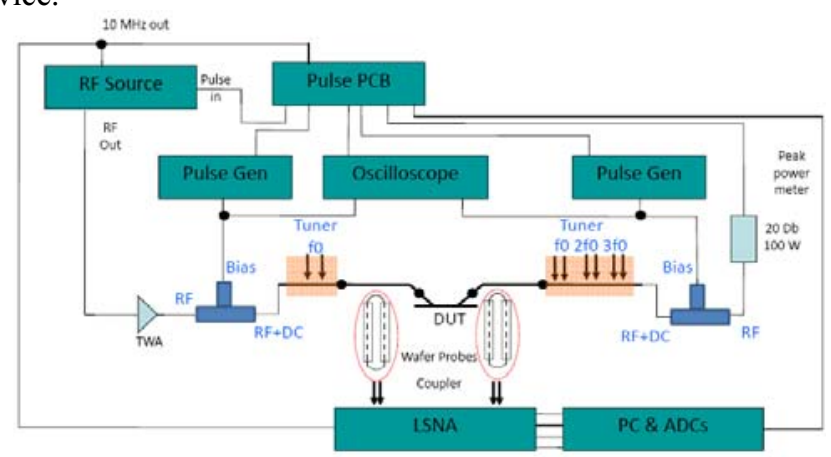

Figure 1. Schematic of the time domain load pull system organized around a Large signal network analyzer (LSNA). Pulse configuration can be managed through a special pulse PCB generator.

\section{B. Enveloppe Measurement System}

The Envelope measurement system represented in Fig-2 was designed for the analysis of the behavior of Power Amplifiers under modulated signal excitation [14]. The base band modulation format is generated by the computer controlling the whole set up and loaded in I / Q ports of the signal modulation unit. The output RF signal of the signal modulation unit is linearly amplified and feeds the input of the power amplifier under test .Time domain complex envelopes of RF signals at the input and the output of the amplifier under test are measured by using vector signal analyzers.

The complex envelope of the RF signal at the PA output is measured by using a Vector Signal analyzer. The magnitude of this complex envelope is also measured at the video out access of the vector signal analyzer and recorded on channel 3 of the scope. Channels 1 and 2 of the scope are used to record drain bias current and voltage. The vector signal analyzer provides the $10 \mathrm{MHz}$ synchronisation signal to the signal modulation unit. The signal modulation unit provides the appropriate trigger signal to the scope and the vector signal analyzer

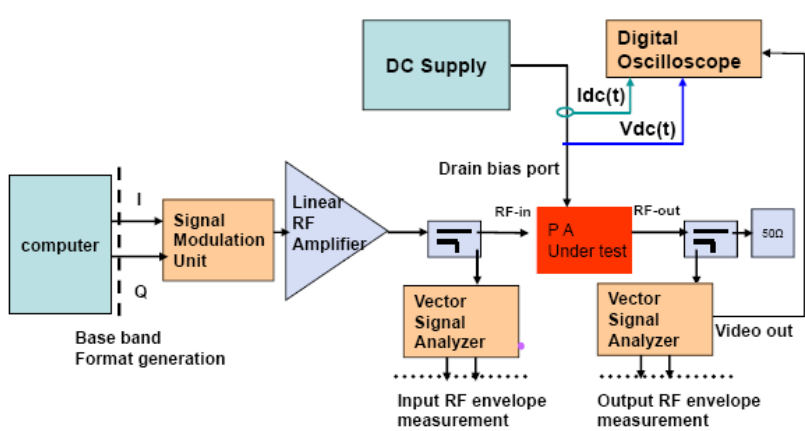

Figure 2. Schematic of the Envelope Measurement system used either for pulsed measurements or for intermodulation measurements.

\section{1) Low-frequency S-parameters measurements}

A third measurement set-up has been used in order to characterize the low frequency dispersive effects. This set combines two Vector Network Analysers (VNA) in order to cover the whole frequency range from $10 \mathrm{~Hz}$ to $40 \mathrm{GHz}$ [15]. This allows measuring the small signal characteristics of the transistors in the frequency domain where parasitic effect take place. Both the thermal and trapping effects have a strong impact on the Low Frequency (LF) characteristics. In the case of Hetrojunction Bipolar Transistors (HBT) where trapping effects are negligible the LF S-parameters allow a precise and full determination of the dynamic thermal behavior of HBT's. In the case of AlGaN/GaN HEMTs the situation is more complex because of the mix of thermal and trapping effects in the same range of frequency. A special care has been dedicated to the development of bias tees able to go down to $10 \mathrm{~Hz}$.

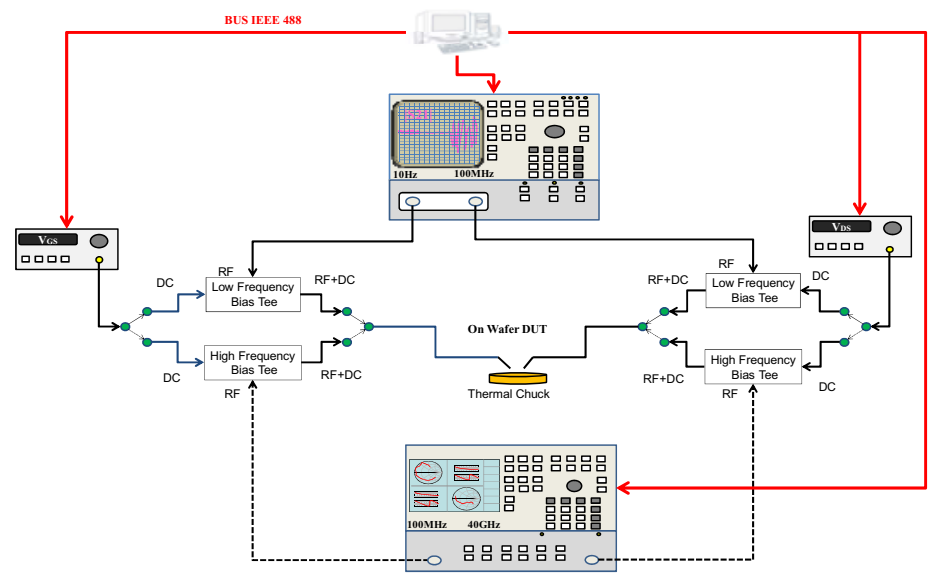

Figure 3. Schematic of the test bench for $10 \mathrm{~Hz}-40 \mathrm{GHz}$ characterization of RF transistors 


\section{RESULTS}

\section{A. Thermal analysis of HBTs through LF S-parameters}

Thermal analysis of $\mathrm{GaAs} / \mathrm{GaInP}$ was performed through LF S-parameters measurements. Indeed it has been shown in [16] that the LF feedback coefficient of the HBTs is only due to the thermal feedback as far as the feedback due to the base collector capacitance is sufficiently low which is the case at LF. In fig.4, the impact of the thermal feedback for a $\mathrm{GaInP} / \mathrm{GaAs} \mathrm{HBT}$ is illustrated by comparing the $\mathrm{S}_{12}$ parameter with and without thermal effects in a nonlinear electrothermal model and with measurements. From this comparison it appears that, at LF, the feedback results only from the thermal behavior.

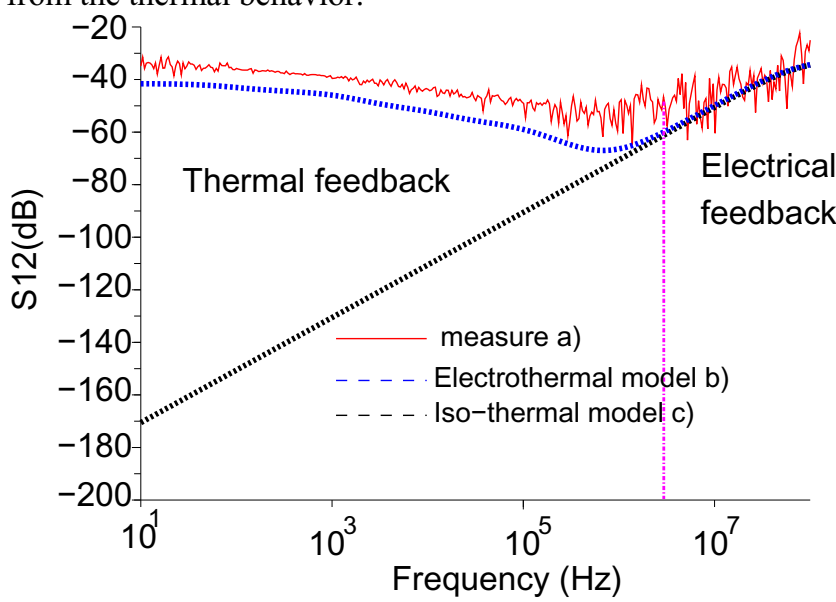

Figure 4. S12 parameter versus frequency for the transistor HBT

$3 \times 2 \times 2 \times 110 \mu \mathrm{m}^{2}$ showing the thermal feedback. Measured S12 a).

Simulated $\mathrm{S} 12$ with an electrothermal model and $\mathrm{Z}_{\mathrm{TH}}$ computed from $3 \mathrm{D}$

finite element simulation $b$ ). Simulated $S 12$ from the model and $Z_{\mathrm{TH}}$ set to zero c).

Moreover, it can be shown [16] that the thermal impedance can be readily extracted from those measurements by transforming the S-parameters in the wellknown hybrid H-parameters by using the first order approximation:

$$
Z_{T H} \cong \frac{h_{12}(\omega)}{\varphi I_{C 0}}
$$

Where $\varphi$ is the base-emitter thermal coefficient $\varphi=\partial \mathrm{Vbe} /\left.\partial \mathrm{T}\right|_{\mathrm{Ib}}$ and $\mathrm{I}_{\mathrm{co}}$ is the bias collector current.

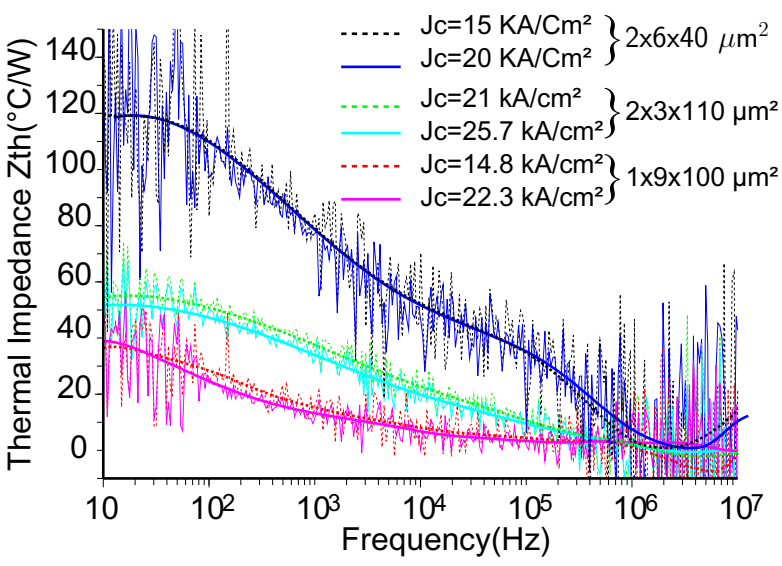

Figure 5. Real Part of thermal impedance versus frequency for different values of collector current density and various sizes of HBTs. As the temperature rise is assumed linear, the thermal impedance is directly related to the temperature of the device times the dissipated power. Measurements are shown here without averaging and the lines are obtained through measurements fitting.

This method has also the advantage of being independent of the size of the transistor and has been used for the determination of the thermal impedance of S-band HBTs as shown in fig-5. Unfortunately it cannot be applied to AlGaN/GaN HEMTs due to the presence of trapping effects.

\section{B. Small signal Frequency dispersion of AlGaN/GaN HEMTS}

In the case of AlGaN/GaN HEMTs the LF S-parameters reveal the trapping effects in much more pronounced fashion than thermal effects. Those measurements like the one shown in fig- 6 allow to better determine the time constants associated with those trapping effects in a model like the one proposed in [18]. However examination of the curves given in fig- 6 shows that those time constants are highly dependent to the drain voltage bias. To our knowledge there is no model able to take this behavior into account yet. Nonlinear models that take into account the trapping effects must be able to fit those characteristics in order to be consistent with all kinds of measurements that can be performed on transistors such as RF pulsed measurements or two tone intermodulation measurements.

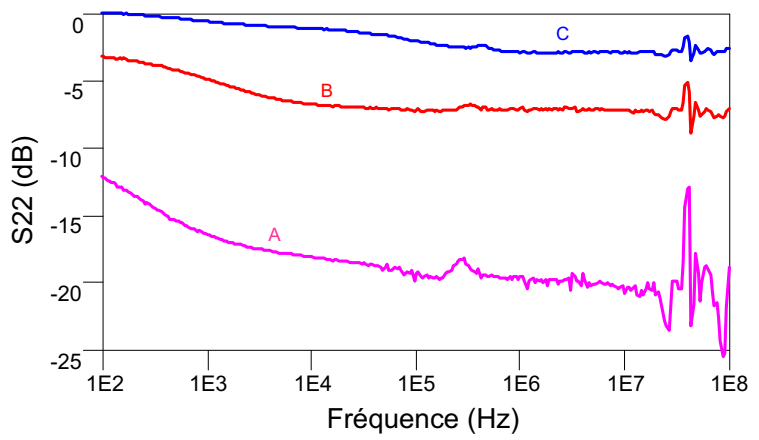


(a)

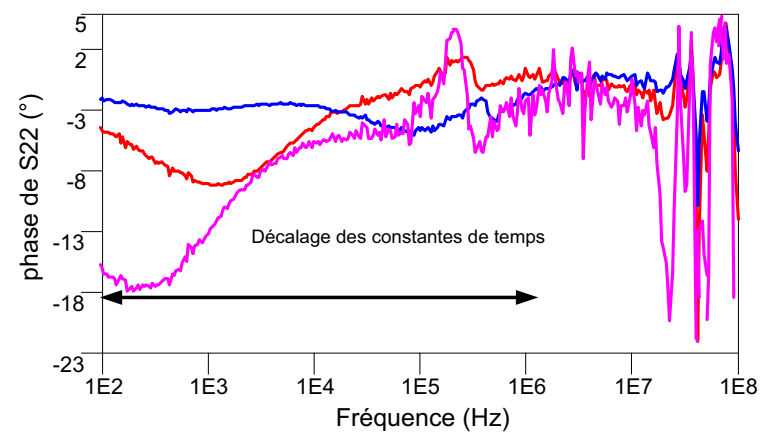

(b)

Figure 6. $\mathrm{S}_{22}$ parameter : (a) magnitude, (b) phase of a transistor $\mathrm{AlGaN} / \mathrm{GaN} 8 \times 75 \mu \mathrm{m}$ at $25^{\circ} \mathrm{C}$ for $\mathrm{Vgs}=-1 \mathrm{~V}$ and $\mathrm{Vds}=4 \mathrm{~V}$ (A) ; $8 \mathrm{~V}$ (B) and $\mathrm{Vds}=20 \mathrm{~V}(\mathrm{C})$

\section{RF Pulsed measurements of AlGaN/GaN HEMTS}

RF Pulsed measurements were performed under DC bias on a $\mathrm{AlGaN} / \mathrm{GaN}$ HEMT of $8 \times 75 \times 0.25 \mu \mathrm{m}^{2}$ from UMS foundry and for a large number of output loads. The shape of the average draint current is shown in Fig-3 for an output load : Zload $=0.467 *$ ej $34.47^{\circ}$ Zload(2fo) $=50$ Ohms. The decrease of this current is due to the trap capture which increases as the gate and drain voltage excursions increase with Pin as explained in [18] for a CW measurement. In this case the RF power was pulsed and the average drain currents exhibit transients corresponding to the capture and emission of traps. For example if the input power is pulsed to $0 \mathrm{dBm}$ the current decreases $\left(\Delta \mathrm{I}_{\mathrm{DT}}\right)$ during the pulse due to the capture of traps. At the end of the pulse, when the input power is switched off, there is a discontinuity in the drain current corresponding to the amount of increase $\left(\Delta \mathrm{I}_{\mathrm{D} 1}\right)$ of the average drain current which should have appeared in the absence of traps as shown in Fig-7-a. Then the captured carriers are re-emitted and the drain current recovers at its bias level. It can be seen that the time constant for emission is of the order of ms while this of capture is well below $10 \mu \mathrm{s}$. In the Fig-7-b it can also be observed that depending of the input power the drain current can increase or decrease. Measurements have also been performed at different duration and recurrence of pulses. They will be presented during the talk.

\section{Two tone measurements}

Same kinds of experiments have been carried out on a $10 \mathrm{~W}$ $\mathrm{GaN}$ power amplifier from Cree (CGH40010F-TB). In pulsed conditions this power amplifier exhibited the same drain current behaviour. In order to assess the impact of traps on linearity and memory characteristics, two-tone measurements have been carried out. The carrier frequency was 3.6 GHz. A sine wave envelope modulation fed the I port of the I/Q modulator while Q access was set to 0 . The frequency of this sine wave envelope modulation was swept from $10 \mathrm{~Hz}$ to $100 \mathrm{KHz}$. This resulted in two tone RF stimuli with various tone spacing at the RF input of the power amplifier under test. Current and voltage probes connected to a sampling scope are used for voltage and current measurements at the drain bias port of the amplifier under test. Measurement results are shown in Fig 8-a and Fig-8-b. The DC bias current is close to $180 \mathrm{~mA}$ and the minimum observed in Fig-4-a corresponds to the instant at which the envelope of the RF wave is equal to zero. In the absence of traps the current should have its bias level. While this is true for very low frequency spacing of the tones, it is no longer true when the frequency spacing increase or, equivalently, when the period of the envelope decreases. In the latter case trap capture occurs when the envelope is at its maximum and the emission time constant is so large that carriers can't be detrapped during the decrease of the envelope of the signal. This results in a minimum current (corresponding to the zero envelope) which is well below the bias level. It must also be noted that the decrease of this minimum is more pronounced as the output power increases.

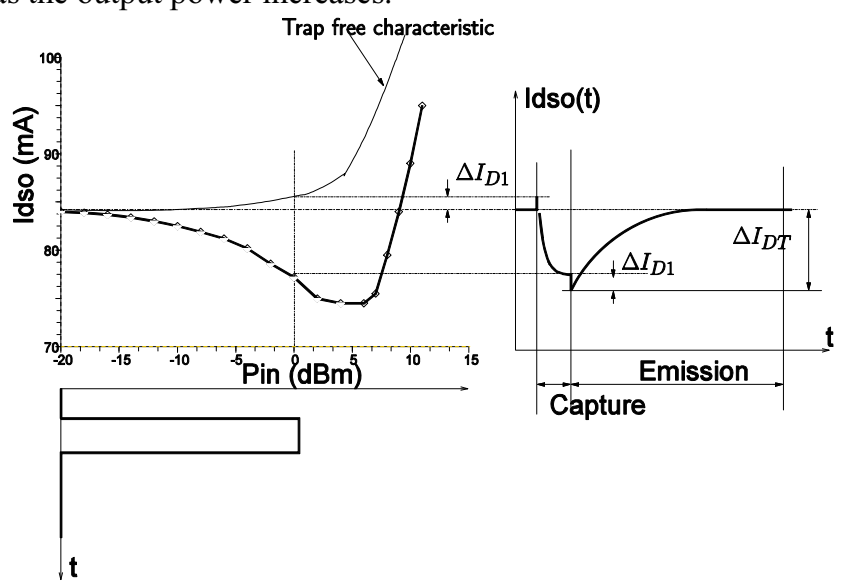

a)

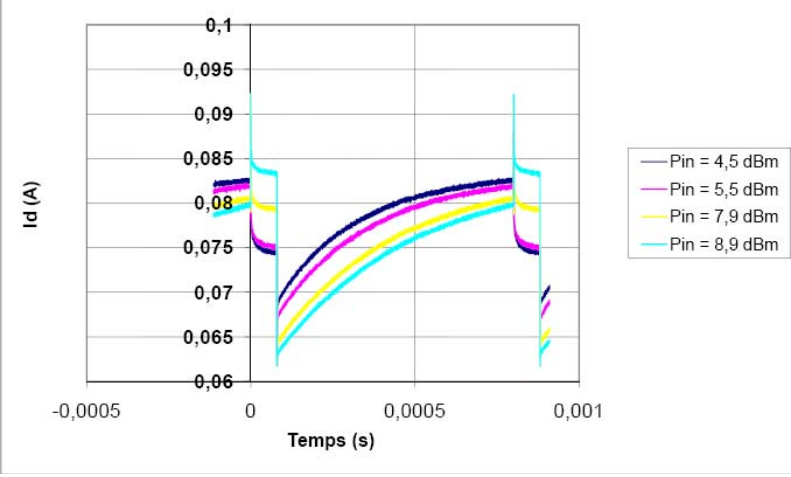

b)

Figure 7. a) Explanation of the shape of the average drain current during an RF pulse. b) Measurements of the average drain current at different power levels. The $8 \times 75 \times 0.25 \mu \mathrm{m}^{2} \mathrm{AlGaN} / \mathrm{GaN}$ HEMT was biased at $\mathrm{Vdso}=30 \mathrm{~V}$ and $\mathrm{Idso}=84 \mathrm{~mA}$. The output load was Zload $=0.467 * \mathrm{ej} 34.47^{\circ}$ Zload $(2 \mathrm{fo})=50 \mathrm{Ohms}$. 


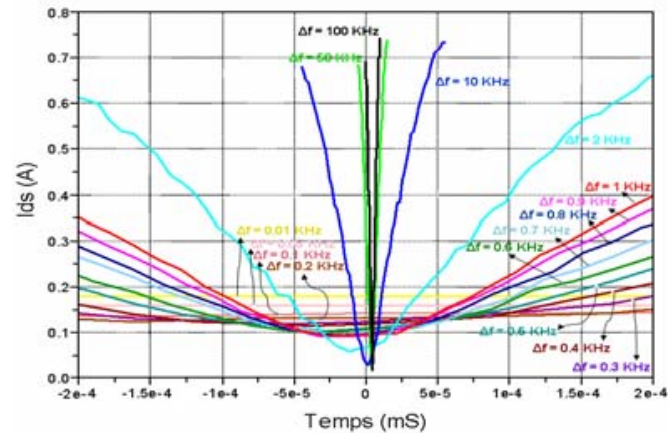

a)

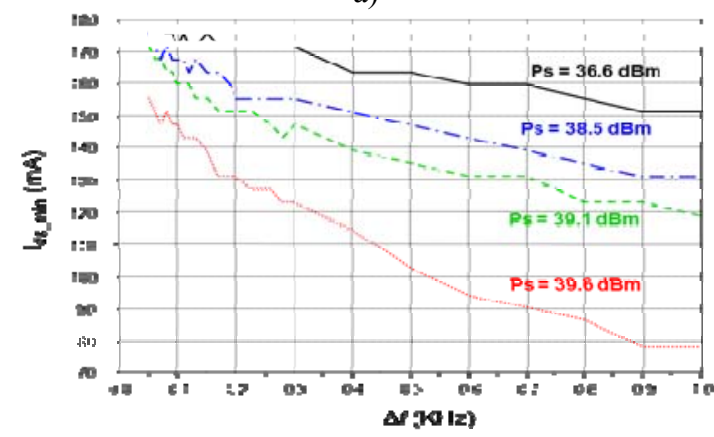

b)

Figure 8. Time domain average drain current of the PA fed by two-tones RF excitation. a) time variation of the current when the frequency spacing is swept from $10 \mathrm{~Hz}$ to $100 \mathrm{KHz}, \mathrm{b}$ ) Variation of the minimum of the current versus frequency at different output power levels.

\section{CONCLUSION}

A number of experiments have been carried out to assess the impact of parasitic effects on output waveforms of power transistors fed by modulated signals - pulses and two-tones-. It has been shown that traps have a significant impact on average drain current in these two cases. Further investigation are currently on going to correlate those behaviors with models extracted from pulsed, LF and RF measurements.

\section{REFERENCES}

[1] D. V. Lang, "Deep-level transient spectroscopy: A new method to characterize traps in semiconductors," Journal of Applied Physics, vol. 45, pp. 3023-3032, 1974.

[2] T. M. Barton, C. M. Snowden, J. R. Richardson, and P. H. Ladbrooke, "Narrow pulse measurement of drain characteristics of GaAs MESFETs," Electronics Letters, vol. 23, pp. 686-687, 1987.

[3] C. Charbonniaud, J. P. Teyssier, and R. Quere, "Time-domain pulsed large-signal non-linear characterization of microwave transistors," in Microwave Conference, 2003. 33rd European, 2003, pp. 241-244 Vol.1.

[4] P. C. Canfield, S. C. F. Lam, and D. J. Allstot, "Modeling of frequency and temperature effects in GaAs MESFETs," Solid-State Circuits, IEEE Journal of, vol. 25, pp. 299-306, 1990.

[5] O. Mitrofanov and M. Manfra, "Mechanisms of Gate-lag in GaN/AlGaN/GaN High Electron Mobility Transistors," Superlattices and Microstructures, vol. 34, pp. 33-53, 2003.
[6] L. L. Peng, P. C. Canfield, and D. J. Allstot, "Trap effects in pchannel GaAs MESFET's," Electron Devices, IEEE Transactions on, vol. 39, pp. 2444-2451, 1992.

[7] K. Kunihiro and Y. Ohno, "A large-signal equivalent circuit model for substrate-induced drain-lag phenomena in HJFETs," Electron Devices, IEEE Transactions on, vol. 43, pp. 1336-1342, 1996.

[8] R. E. Leoni, III, J. W. Bao, X. Du, M. Shirokov, and J. C. M. Hwang, "Conductance DLTS analysis of the correlation between power slump and gate lag," in Gallium Arsenide Integrated Circuit (GaAs IC) Symposium, 1997. Technical Digest 1997., 19th Annual, 1997, pp. 169-172.

[9] Z. Ouarch, J. M. Collantes, J. P. Teyssier, and R. Quere, "Measurement based nonlinear electrothermal modeling of GaAs FET with dynamical trapping effects," in Microwave Symposium Digest, 1998 IEEE MTT-S International, 1998, pp. 599-602 vol.2.

[10] M. S. Shirokov, R. E. Leoni, III, J. Bao, and J. C. M. Hwang, "A transient SPICE model for digitally modulated RF characteristics of ion-implanted GaAs MESFET's," Electron Devices, IEEE Transactions on, vol. 47, pp. 1680-1681, 2000.

[11] N. Matsunaga, M. Yamamoto, Y. Hatta, and H. Masuda, "An improved GaAs device model for the simulation of analog integrated circuit," Electron Devices, IEEE Transactions on, vol. 50, pp. 11941199,2003

[12] F. De Groote, J. P. Teyssier, O. Jardel, T. Gasseling, and J. Verspecht, "Introduction to measurements for power transistor characterization," Microwave Magazine, IEEE, vol. 9, pp. 70-85, 2008.

[13] J. Faraj, G. Callet, F. De Groote, J. Verspecht, R. Quere, and J. P. Teyssier, "Bursts of Pulses for time domain large signal measurements," in Microwave Measurement Conference, 2009 73rd ARFTG, 2009, pp. 1-4.

[14] M. Saad El Dine, T. Reveyrand, G. Neveux, P. Bouysse, D. Barataud, J. M. Nébus, and W. Rebernack, "A Measurement Set-up and Methodology Combining Dynamic Biasing and Baseband Predistorsion for High Efficiency and Linear Amplifier Design," in IEEE International Microwave Symposium, Anaheim, California, USA, 2010, pp. 1070-1073.

[15] A. El Rafei, G. Callet, G. Mouginot, J. Faraj, S. Laurent, M. Prigent, R. Quere, O. Jardel, and S. Delage, "DC $(10 \mathrm{~Hz})$ to RF $(40 \mathrm{GHz})$ output conduction extraction by S-parameters measurements for indepth characterization of AlInN/GaN HEMTS, focusing on low frequency dispersion effects," in Microwave Integrated Circuits Conference (EuMIC), 2011 European, 2011, pp. 5-8.

[16] A. Xiong, R. Sommet, A. A. L. de Souza, and R. Quere, "Dual approach for HBT thermal impedance," in Thermal Inveatigation of ICs and Systems, 2008. THERMINIC 2008. 14th International Workshop on, 2008, pp. 190-194.

[17] A. El Rafei, R. Sommet, and R. Quere, "Electrical Measurement of the Thermal Impedance of Bipolar Transistors," Electron Device Letters, IEEE, vol. 31, pp. 939-941, 2010.

[18] O. Jardel, F. De Groote, T. Reveyrand, J. C. Jacquet, C. Charbonniaud, J. P. Teyssier, D. Floriot, and R. Quere, "An electrothermal model for $\mathrm{AlGaN} / \mathrm{GaN}$ power HEMTs including trapping effects to improve large-signal simulation results on high VSWR," IEEE Transactions on Microwave Theory and Techniques, vol. 55, pp. 2660-2669, Décembre 2007. 